\title{
Surface subgroups of Mapping Class groups
}

\author{
Alan W. Reid * \\ March 4, 2005
}

\section{Introduction}

Let $\Sigma$ be a compact oriented surface, possibly with boundary, the Mapping Class group of $\Sigma$ is

$$
\operatorname{Mod}(\Sigma)=\operatorname{Homeo}^{+}(\Sigma) / \operatorname{Homeo}_{0}(\Sigma)
$$

where, $\mathrm{Homeo}^{+}(\Sigma)$ is the group of orientation preserving homeomorphisms of $\Sigma$ and $\mathrm{Homeo}_{0}(\Sigma)$ are those homeomorphisms isotopic to the identity. When $\Sigma$ is a closed surface of genus $g \geq 1$ then we denote $\operatorname{Mod}(\Sigma)$ by $\Gamma_{g}$. In this case it is well-known that $\Gamma_{g}$ is isomorphic to a subgroup of index 2 in $\operatorname{Out}\left(\pi_{1}(\Sigma)\right)$.

When $g=1$, the subgroup structure of $\Gamma_{1}$ is well-understood since it is simply the group $\mathrm{SL}(2, \mathbf{Z})$. When $g \geq 2$, attempts to understand the subgroup structure of $\Gamma_{g}$ have been made by exploiting the many analogies between $\Gamma_{g}$ and non-uniform lattices in Lie groups. In particular, both the finite and infinite index subgroup structure of $\Gamma_{g}$ has many parallels in the theory of lattices. For example, the question of whether Property $\mathrm{T}$ holds for $\Gamma_{g} \geq 3$ (it fails for $g=2$ by [29]), whether $\Gamma_{g}$ has a version of the Congruence Subgroup Property, or towards the other extreme, whether there are finite index subgroups of $\Gamma_{g}$ that surject onto $\mathbf{Z}$ (see for example [11], [16], [18] and [29] for more on these directions). The discussion and questions raised in this paper are motivated by analogies between the subgroup structure of $\Gamma_{g}$ and non-cocompact but finite co-volume Kleinian groups. To that end, we are particularly interested in the nature of surface subgroups of $\Gamma_{g}$. For an exploration of other analogies between $\Gamma_{g}$ and Kleinian groups see [13], [19] and [33].

Throughout, the terms surface (sub)group will be reserved for $\pi_{1}\left(\Sigma_{g}\right)$ where $g \geq 2$, and a subgroup of $\Gamma_{g}$ is said to be purely pseudo-Anosov if all non-trivial elements are pseudo-Anosov. Simply put our motivation is the following question.

Question 1.1 For $g \geq 2$, does $\Gamma_{g}$ contain a purely pseudo-Anosov surface subgroup?

The paper is organized as follows. In $\S 2$, we discuss the existence of surface subgroups in Kleinian groups of finite co-volume. In $\S 3$, we discuss surface subgroups of $\Gamma_{g}$, and contrast and compare with $\S 2$. In $\S 4$ we discuss some related topics; for example we discuss the connection of Question 1.1 with some conjectures in 4-manifold topology.

Acknowledgements: Much of the content of this paper is related to joint work of the author and Chris Leininger [23], and the author gratefully acknowledges his contributions to this work. In addition, some of the questions mentioned here also appear in [23]. The author would also like to thank Ian Agol, Richard Kent and Darren Long for useful conversations on some of the topics discussed here.

*This work was partially supported by an N.S.F. grant 


\section{Surface subgroups of Kleinian groups}

We begin by discussing the case of surface subgroups in Kleinian groups; see [26], [37], or [32] for terminolgy.

\section{1}

A Kleinian group $\Gamma$ is a discrete subgroup of $\operatorname{PSL}(2, \mathbf{C})$, and as such, $\Gamma$ acts discontinuously on $\mathbf{H}^{3}$ and the quotient $\mathbf{H}^{3} / \Gamma$ is a hyperbolic 3-orbifold. When $\Gamma$ is torsion-free, $\mathbf{H}^{3} / \Gamma$ is a hyperbolic 3 -manifold. Of interest to us is the case when $\mathbf{H}^{3} / \Gamma$ is closed or finite volume.

Let $\Sigma$ be a closed orientable surface of genus at least $2, M=\mathbf{H}^{3} / \Gamma$ an orientable finite volume hyperbolic 3-manifold and $f: \Sigma \rightarrow M$ a map. We shall call $f(\Sigma)$ (or by abuse simply $\Sigma$ ) an essential surface if $f_{*}: \pi_{1}(\Sigma) \rightarrow \Gamma$ is injective. This is non-standard terminology, but will be convenient for our purposes. If $M$ contains an essential surface then $\Gamma$ contains a surface subgroup. The converse is also true, and given this, an important question in 3-manifold topology is:

Question 2.1 Let $M=\mathbf{H}^{3} / \Gamma$ be a finite volume hyperbolic 3-manifold, does $\Gamma$ contain a surface subgroup.

This was answered in [8] for non-compact but finite volume manifolds, and so the remaining cases of Question 2.1 are the closed manifolds, and this seems far from resolution at present. We will discuss [8] in more detail below.

\section{2}

Given an essential surface $\Sigma$ in a finite volume hyperbolic 3-manifold $M$, one can attempt to understand the surface in terms of how the hyperbolic metric on $M$ restricts to the surface $\Sigma$. In a non-compact finite volume hyperbolic 3-manifold there are two possibilities for the geometry of a closed essential surface $\Sigma . \Sigma$ is either quasi-Fuchsian or it is said to contain accidental parabolics. In the latter case, as suggested by the name, these surface groups contain parabolic elements, whilst in the former case, all non-trivial elements are hyperbolic. Both these surfaces are geometrically finite. The methods of [8] only provide surfaces containing accidental parabolics, and a comment on the construction in [8] will be informative for our discussion of $\Gamma_{g}$ (see also [7]).

Suppose that $M=\mathbf{H}^{3} / \Gamma$ is a non-compact finite volume hyperbolic 3-manifold. We can assume that $M$ is orientable on passing to a double cover if necessary, and so $M$ is the interior of a compact 3 -manifold with boundary consisting of a disjoint union of tori. Using residual finiteness of $\Gamma$ there is a finite cover, $\hat{M}$ of $M$, which has at least 3 boundary components. Standard 3-manifold topology shows that the first betti number of $\hat{M}$ is at least 3 , and that one can find an embedded orientable essential surface $F$ with non-empty boundary in $\hat{M}$ missing at least one of the boundary components of $\hat{M}$. This surface can then be used to build a finite cyclic cover of $\hat{M}$ containing a closed embedded essential surface $\Sigma$ of genus at least 2. Indeed, the construction is explicit, the surface $\Sigma$ is constructed by taking two copies of $F$ "tubed together" along their boundary. $\Sigma$ pushes down to $M$ to provide an essential surface in $M$ and so the desired surface subgroup. By construction, these "tubed surfaces" contain accidental parabolic elements corresponding to the peripheral elements from $\partial F$.

The question of existence of closed quasi-Fuchsian surfaces in $M$ as above remains open. However recent work of Masters and Zhang [27] appears to make some progress on this. 


\section{3}

The surface subgroups built in [8] can also be viewed as being built by repeated applications of the Maskit combination theorems, a version of which is given below (see [23] and [26] Chapter VII for more details).

Suppose $G_{0}, G_{1}, G_{2}<\Gamma$ with $G_{1} \cap G_{2}=G_{0}<\Gamma$. If $\Gamma$ acts on a set $X$, then we say that a pair of subsets $\Theta_{1}, \Theta_{2} \subset X$ is a proper interactive pair for $G_{1}, G_{2}$ if

1. $\Theta_{i} \neq \emptyset$ for each $i=1,2$,

2. $\Theta_{1} \cap \Theta_{2}=\emptyset$,

3. $G_{0}$ leaves $\Theta_{i}$ invariant for each $i=1,2$,

4. for every $\phi_{1} \in G_{1} \backslash G_{0}$ we have $\phi_{1}\left(\Theta_{2}\right) \subset \Theta_{1}$ and for every $\phi_{2} \in G_{2} \backslash G_{0}$ we have $\phi_{2}\left(\Theta_{1}\right) \subset \Theta_{2}$, and

5. for $i=1,2$, there exists $\theta_{i} \in \Theta_{i}$, such that for every $\phi_{i} \in G_{i} \backslash G_{0}, \theta_{i} \notin \phi_{i}\left(\Theta_{i^{\prime}}\right)$ for $i^{\prime} \neq i$.

With this notation, we can state the following combination theorem.

Theorem 2.2 Suppose $G_{0}, G_{1}, G_{2}, \Gamma, X$ are as above and $\Theta_{1}, \Theta_{2} \subset X$ is a proper interactive pair for $G_{1}, G_{2}$. Then

$$
G=G_{1} *_{G_{0}} G_{2} \hookrightarrow \Gamma
$$

is an injection.

The construction of essential surfaces in non-compact finite volume hyperbolic 3-manifolds discussed in $\S 2.2$ can also be described using Theorem 2.2 (and an HNN version of it) applied to the two copies of the surface $F$. In the particular case when the surface $F$ has only one boundary component, the closed surface $D F$ constructed by the above has the property that all non-trivial elements are hyperbolic except those conjugate into the cyclic subgroup generated by the parabolic element $\alpha=[\partial F]$ in $\pi_{1}(D F)$.

\section{4}

The dichotomy of closed quasi-Fuchsian versus those that contain an accidental parabolic also manifests itself in the structure of surface subgroups of $\Gamma$. Using the compactness of pleated surfaces, Thurston shows in [37] that for a fixed genus $g$ there are only finitely many $\Gamma$-conjugacy classes of quasi-Fuchsian surface subgroups of $\Gamma$ of genus $g$. On the otherhand, given an essential surface $\Sigma$ of genus $g$ in $M$ with an accidental parabolic it is easy to construct infinitely many conjugacy classes of surface subgroups of genus $g$ (see [37] Chapter 8).

\section{Surface subgroups in $\Gamma_{g}$}

Throughout this section we assume that $\Sigma$ is a closed oriented surface of genus $g \geq 2$. We will denote the Teichmüller space of $\Sigma$ by $\mathcal{T}(\Sigma)$, the space of compactly supported measured laminations by $\mathcal{M L}_{0}(\Sigma)$, and $\mathbb{P} \mathcal{M} \mathcal{L}_{0}(\Sigma)$ the space of projective measured laminations on $\Sigma$. 


\section{1}

Given any element $\phi \in \Gamma_{g}$, one of the following holds; $\phi$ has finite order, $\phi$ is reducible, or $\phi$ is pseudo-Anosov. An infinite order element $\phi \in \Gamma_{g} \backslash\{1\}$ is pseudo-Anosov if there exists a pair of measured laminations $\lambda_{s}, \lambda_{u}$ which bind $\Sigma$ and such that $\left\{\left[\lambda_{s}\right],\left[\lambda_{u}\right]\right\}$ is invariant by $\phi$. An element $\phi \in \Gamma_{g} \backslash\{1\}$ is reducible if there exists a multi-curve (ie a finite union of essential simple closed curves on $\Sigma$ which are pairwise disjoint and parallel) invariant by $\phi$.

If $q$ is holomorphic quadratic differential on $\Sigma$, then for any constant, nonzero 1-form $a d x+b d y$ on $\mathbf{C}(a, b \in \mathbf{R})$, the measured foliation $|a d x+b d y|$ is invariant under the transition functions for the atlas of $q$-coordinates, and so pulls back to a measured foliation on the complement of the cone points. This defines a singular measured foliation on $\Sigma$ and the space of all such measured foliations is denoted $\mathcal{M L}_{0}(q)$.

\section{2}

Although an answer to Question 1.1 remains unknown at present, there appears to be an interesting analogue to the discussion in $\S 2$. It had been known for some time that $\Gamma_{g}$ contained surface subgroups (see [2] and [15] for example), however a more uniform treatment of constructions of surface subgroups is given in [23]. In some sense this can be viewed as an analogue of the results in [8]. To describe this more fully, we require some terminology.

Veech subgroups of $\Gamma_{g}$ arise from stabilizers of Teichmüller discs in the Teichmüller space and have been objects of some interest of late (see [22], [31] and [40] for more on related topics). Briefly, these arise as follows.

Any holomorphic quadratic differential $q$ on $\Sigma$ defines a holomorphic totally geodesic embedding of the hyperbolic plane

$$
f_{q}: \mathbf{H}^{2} \rightarrow \mathbf{H}_{q} \subset \mathcal{T}(\Sigma)
$$

The stabilizer $\operatorname{Stab}_{\Gamma_{g}}\left(\mathbf{H}_{q}\right)$ acts on $\mathbf{H}_{q}$ and this action can be conjugated back to $\mathbf{H}^{2}$, via $f_{q}$, thus defining a homomorphism $D$ to $\operatorname{PSL}(2, \mathbf{R})$. Subgroups of $\operatorname{Stab}_{\Gamma_{g}}\left(\mathbf{H}_{q}\right)$ are called Veech groups. If $\operatorname{PSL}(q)$ denotes the image of $\operatorname{Stab}_{\Gamma_{g}}\left(\mathbf{H}_{q}\right)$ under $D$, then in the special case when $\mathbf{H}^{2} / \operatorname{PSL}(q)$ has finite area, this quotient is called a Teichmüller curve and this immerses into the moduli space. This is our analogue of an immersed essential non-compact surface in a finite volume hyperbolic 3-manifold.

An example we will make use of is the following due to Veech [40]. Let $\Delta_{g}$ be the non-convex polygon obtained as the union of two regular $2 g+1$-gons in the Euclidean plane which meet along an edge and have disjoint interiors. Let $R_{g}$ denote the closed surface of genus $g$ obtained by gluing opposite sides of $\Delta_{g}$ by translations. The Euclidean metric on the interior of $\Delta_{g}$ is the restriction of a Euclidean cone metric on $R_{g}$, and we can find local coordinates defining a quadratic differential on $R_{g}$ compatible with this metric. We denote this quadratic differential $\xi_{g}$, and $F\left(\xi_{g}\right)$ the associated Veech group. Veech showed that $F\left(\xi_{g}\right)$ is isomorphic to a triangle group of type $(2,2 g+1, \infty)$, where the single primitive parabolic conjugacy class in the triangle group corresponds to the conjugacy class of a reducible element $\delta$. It is easy to see that the above triangle group contains as a subgroup of index $2(2 g+1)$ which is the fundamental group of a 1-punctured surface of genus $g$. Hence this determins a subgroup $G\left(\xi_{g}\right)$ of $F\left(\xi_{g}\right)$ isomorphic to the fundamental group of a 1-punctured surface of genus $g$.

A particular case of the main construction of [23], using the Combination Theorem (Theorem 2.2 ) is the following, and which produces examples that are "closest to purely pseudo-Anosov" obtained thus far (cf. the discussion after Theorem 2.2).

Theorem 3.1 For every $g \geq 2$, there exist subgroups of $\Gamma_{g}$ isomorphic to the fundamental group of a closed surface of genus $2 g$. These are obtained as the amalgamated free product of two copies 
of $G\left(\xi_{g}\right)$ along the infinite cyclic subgroup $\langle\delta\rangle$. In addition, all but one conjugacy class of non-trivial elements (up to powers) is pseudo-Anosov.

As in the 3-manifold setting, it can also be shown that there are infinitely many distinct conjugacy classes of surface subgroups of genus $2 g$ (see [23]). Motivated by Thurstons result discussed in $\S 2.4$ we pose:

Question 3.2 Are there are only finitely many $\Gamma_{g}$-conjugacy classes of purely pseudo-Anosov surface subgroups of any fixed genus?

Of course given that Question 1.1 is open, the answer to Question 3.2 could be zero.

\section{3}

As mentioned in $\S 2.2$, quasi-Fuchsian surface subgroups and those with accidental parabolics are geometrically finite. Furthermore, the combination theorem in the Kleinian group setting shows that combining two geometrically finite groups (with some assumption on the amalgamating subgroup) gives a geometrically finite group.

The notion of geometrical finiteness is somewhat more delicate in the setting of subgroups of $\Gamma_{g}$. In [13] the notion of convex cocompactness of subgroups of $\Gamma_{g}$ is given, and an attempt to extend this to geometrically finite is proposed in [33]. In particular, the question of geometrical finiteness of the groups in Theorem 3.1 as well as others constructed in [23] is posed. We refer the reader to [13] and [33] for more on the notions of convex cocompact and geometrically finite subgroups in the context of $\Gamma_{g}$, as well as for questions concerning the geometrical finiteness of various subgroups.

\section{4}

Taking the analogy with hyperbolic spaces further, we recall that Thurston's compactification of Teichmüller space is obtained by adding $\mathbb{P} \mathcal{M} \mathcal{L}_{0}(\Sigma)$ at infinity to obtain

$$
\overline{\mathcal{T}}(\Sigma)=\mathcal{T}(\Sigma) \cup \mathbb{P} \mathcal{M} \mathcal{L}_{0}(\Sigma) \cong B^{6 g-6}
$$

where $B^{6 g-6}$ is the closed ball of dimension $6 g-6$ and $\mathbb{P} \mathcal{M} \mathcal{L}_{0}(\Sigma)$ is identified with the boundary. Moreover, $\mathbb{P} \mathcal{M} \mathcal{L}_{0}(\Sigma)$ has a natural piecewise projective structure and the action of $\Gamma_{g}$ on $\mathcal{T}(\Sigma)$ and $\mathbb{P}_{\mathcal{M}} \mathcal{L}_{0}(\Sigma)$ fit together to give a well defined action on $\overline{\mathcal{T}}(\Sigma)$ which is holomorphic on the interior and piecewise projective on the boundary.

There is a natural identification of $\mathbb{P} \mathcal{M} \mathcal{L}_{0}(q)$ with the boundary at infinity $\partial_{\infty} \mathbf{H}^{2}$. In this way, the inclusion of $\mathbb{P} \mathcal{M} \mathcal{L}_{0}(q)$ into $\mathbb{P} \mathcal{M} \mathcal{L}_{0}(\Sigma)$ can be thought of as an extension $\partial_{\infty} f_{q}$ of $f_{q}$ to infinity. Indeed, the natural projective structure on $\mathbf{R} \mathbb{P}^{1}=\partial_{\infty} \mathbf{H}^{2}=\mathbb{P} \mathcal{M} \mathcal{L}_{0}(q)$ makes $\partial_{\infty} f_{q}$ into a piecewise projective embedding, equivariant with respect to the $\operatorname{Stab}_{\Gamma_{g}}\left(\mathbf{H}_{q}\right)$ action.

Moreover, $\partial_{\infty} f_{q}$ sends the limit set $\Lambda(\operatorname{PSL}(q)) \subset \partial_{\infty} \mathbf{H}^{2}=\mathbb{P} \mathcal{M} \mathcal{L}_{0}(q)$ homeomorphically and $\operatorname{Stab}_{\Gamma_{g}}\left(\mathbf{H}_{q}\right)$-equivariantly to the limit set $\Lambda\left(\operatorname{Stab}_{\Gamma_{g}}\left(\mathbf{H}_{q}\right)\right) \subset \mathbb{P} \mathcal{M} \mathcal{L}_{0}(\Sigma)$ as defined by McCarthy and Papadopoulos [30].

The map

$$
\bar{f}_{q}=f_{q} \cup \partial_{\infty} f_{q}: \overline{\mathbf{H}}^{2}=\mathbf{H}^{2} \cup \mathbb{P} \mathcal{M} \mathcal{L}_{0}(q) \rightarrow \overline{\mathcal{T}}(\Sigma)
$$

is continuous for every $p \in \mathbb{H}^{2}$ and almost every $p \in \mathbb{P} \mathcal{M} \mathcal{L}_{0}(q)$ by a theorem of Masur [28]. However, Masur's theorem implies that this is in general not continuous at every point of $\mathbb{P} \mathcal{M} \mathcal{L}_{0}(q)$.

This now leads us to the following natural question, the analog of which is true in the setting of Kleinian groups. 
Question 3.3 Let $G \cong \pi_{1}\left(S_{2 g}\right) \rightarrow \Gamma_{g}$ be the injection given by Theorem 3.1. Consider $\partial_{\infty}(G)$ which can be canonically identified with the circle at infinity of the universal cover $\widetilde{S}_{2 g} \cong \mathbb{H}^{2}$ of $S_{2 g}$. Does there exist a continuous G-equivariant map

$$
\partial_{\infty}(G) \rightarrow \mathbb{P} \mathcal{M} \mathcal{L}_{0}(\Sigma) ?
$$

\section{5}

In the context of 3-manifold topology, given an essential surface $\Sigma$ in a finite volume hyperbolic 3-manifold $M$, a natural question is whether there is a finite cover of $M$ to which $\Sigma$ lifts to an embedded surface. A group theoretic property that is closely related to this question is LERF, which now define.

If $\Gamma$ is a group, and $H$ a subgroup of $\Gamma$, then $\Gamma$ is called $H$-separable if for every $g \in G \backslash H$, there is a subgroup $K$ of finite index in $\Gamma$ such that $H \subset K$ but $g \notin K$. $\Gamma$ is called LERF or subgroup separable if $\Gamma$ is $H$-separable for all finitely generated subgroups $H$. This has been widely studied in the setting of low-dimensional topology (see [1] and [35] for example). Indeed, it is often the case that one does not need the full power of LERF for applications to hyperbolic manifolds, separating geometrically finite subgroups often suffices; this led to the property of GFERF, that is separable on all geometrically finite subgroups.

Now it is known that the groups $\Gamma_{g}$ are not LERF whenever $g \geq 2$ since they contain $F_{2} \times F_{2}$, however in analogy with the case for hyperbolic manifolds we pose (for $g=1$ it is known that $\Gamma_{1}$ is LERF since it is virtually free):

Question 3.4 Is $\Gamma_{g}$ GFERF for $g \geq 2$ ?

Question 3.5 Let $H$ be a convex cocompact subgroup of $\Gamma_{g}$. Is $\Gamma_{g} H$-separable?

Just focusing on surface subgroups, we can ask:

Question 3.6 Let $H$ be a surface subgroup of $\Gamma_{g}$. Is $\Gamma_{g} H$-separable?

For recent progress on various classes of subgroups of $\Gamma_{g}$ that are separable, we refer the reader to $[24]$.

\section{Related topics}

Although it is widely believed that if $M=\mathbf{H}^{3} / \Gamma$ is non-compact and finite volume, then $\Gamma$ contains a closed quasi-Fuchsian surface subgroup, it is unclear how Question 1.1 will be resolved at present. We next discuss some related questions, that may help shed light on it.

\section{1}

One motivation for Question 1.1 is the following question from 4-manifold topology.

Question 4.1 Does there exist a closed hyperbolic 4-manifold $X$ that is the total space of a smooth fiber bundle $\Sigma_{g} \rightarrow X \rightarrow \Sigma_{h}$ ?

We will call such an $X$ a surface bundle over a surface. The following is well-known.

Theorem 4.2 A postive answer to Question 4.1 implies a positive answer to Question 1.1 
Proof: Let $X$ be a closed hyperbolic 4-manifold that is a surface bundle over a surface. Then the long homotopy exact sequence defines $\pi_{1}(X)$ as a short exact sequence:

$$
1 \rightarrow \pi_{1}\left(\Sigma_{g}\right) \rightarrow \pi_{1}(X) \rightarrow \pi_{1}\left(\Sigma_{h}\right) \rightarrow 1
$$

and therefore defines a homomorphism $\phi: \pi_{1}\left(\Sigma_{h}\right) \rightarrow \Gamma_{g}$. Indeed, since $X$ is hyperbolic, it follows that $\phi$ is injective. Furthermore the image of $\phi$ must be purely pseudo-Anosov. For if not, since $\pi_{1}(X)$ is torsion-free, there is a reducible element $\psi$ in the image. Let $M_{\psi}$ denote the 3-manifold fibered over the circle with fiber $\Sigma_{g}$ and monodromy $\psi$. From the injection of $\pi_{1}\left(\Sigma_{h}\right)$ into $\Gamma_{g}$ it follows that $\pi_{1}\left(M_{\psi}\right)$ injects into $\pi_{1}(X)$. Now reducibility implies that $\pi_{1}\left(M_{\psi}\right)$ contains a copy of $\mathbf{Z} \oplus \mathbf{Z}$, but $X$ is a closed hyperbolic 4-manifold and contains no such subgroup.

At present there seems little about the geometry and topology of hyperbolic 4-manifolds that can be brought to bear on this question. However, two trivial remarks are:

1. With $X$ as above then the signature of $X$ is 0 .

In fact this holds more generally for any closed hyperbolic 4-manifold; since any hyperbolic 4manifold is conformally flat, so the Hirzebruch signature theorem implies that the signature is zero.

2. The hyperbolic volume of $X$ is

$$
\operatorname{Vol}(X)=\frac{4 \pi^{2}}{3} \chi\left(\Sigma_{g}\right) \chi\left(\Sigma_{h}\right)
$$

This follows from the observations that for any finite volume hyperbolic 4-manifold $M, \operatorname{Vol}(M)=$ $\frac{4 \pi^{2}}{3} \chi(M)$, and for $X$ a surface bundle over a surface with base $\Sigma_{h}$ and fiber $\Sigma_{g}$, we have $\chi(X)=$ $\chi\left(\Sigma_{g}\right) \chi\left(\Sigma_{h}\right)$.

Note that the volume formula shows quickly that the genus of both the base and fiber in this setting is at least 2. Also, using Wang's finiteness result [41] on the finiteness of the number of isometry classes of hyperbolic manifolds of a fixed volume, it follows from this second remark that for fixed $g, h \geq 2$, there are only finitely many manifolds $X$ as above with fiber of genus $g$ and base of genus $h$.

Given that the smallest known Euler characteristic of a closed orientable hyperbolic 4-manifold is 16 [6], a natural warm-up question might be.

Question 4.3 Does there exist a closed hyperbolic 4-manifold that is a surface bundle over a surface where the genus of the fiber and base is 2 ?

\section{2}

Some evidence for a negative answer to Question 4.1 is given in [21] where the following conjecture is stated (this is a special case of a more general conjecture on vanishing of Seiberg-Witten invariants). We refer to [36] and [14] for definitions.

Conjecture 4.4 Let $M$ be a closed hyperbolic 4-manifold. Then all the Seiberg-Witten invariants of $M$ vanish.

The relevance of this is given in the following proposition. We give a proof for hyperbolic manifolds that makes use only of Taubes celebrated paper [36] when $b_{2}^{+}>1$, and hence avoid complexities that arise when $b_{2}^{+}=1$. 
Proposition 4.5 A postive answer to Conjecture 4.4 implies that a closed hyperbolic 4-manifold $M$ cannot be symplectic.

Proof: This follows automatically from [36] in the case when $b_{2}^{+}>1$, since [36] shows that a compact oriented symplectic 4-manifold with $b_{2}^{+}>1$ has non-vanishing Seiberg-Witten invariants. Thus assume that $b_{2}^{+}(M)=1$, and $M$ is symplectic (if $b_{2}(M)=0$ there is nothing to prove). From the discussion above, since $M$ is hyperbolic, the signature of $M$ is 0 , and so $b_{2}(M)=2$. Also $M$ being hyperbolic implies $\pi_{1}(M)$ is residually finite and so $M$ has many finite covers. Let $p: M_{1} \rightarrow M$ be a cover of degree $d>1$. Since $M$ is symplectic, $M_{1}$ will be symplectic using the pullback of the symplectic form on $M$. We claim that $b_{2}^{+}\left(M_{1}\right)>1$, and so we can apply [36] to get a contradiction.

For if $b_{2}\left(M_{1}\right)=2$, then (from the volume formula above) since the Euler characteristic satisfies $\chi\left(M_{1}\right)>0$, it follows that $b_{1}\left(M_{1}\right)<2$. However, $\chi\left(M_{1}\right)=d \chi(M)$ and this shows $b_{1}\left(M_{1}\right)=$ $2-d\left(2-b_{1}(M)\right)$. These remarks yield the desired contradiction.

On the otherhand, any $X$ that has the description of a surface bundle over a surface where both the base and fiber have genus $\geq 2$ is symplectic by an old argument of Thurston (see [38] or [14] Theorem 10.2.17).

Motivated by this discussion, one can ask the following generalization of Question 4.1, which is an analogue of the virtual fibering question in dimension 3 .

Question 4.6 Is every finite volume hyperbolic 4-manifold virtually symplectic; ie has a finite cover that admits a symplectic structure.

We note that it is easy to construct non-symplectic hyperbolic 4-manifolds. For example it is easy to see that the Davis manifold $D$ (see [9]) admits no symplectic structure using the following simple parity rule (see [14] Corollary 10.1.10):

Suppose that $(M, \omega)$ is a closed symplectic 4-manifold. Then $1-b_{1}(M)+b_{2}^{+}(M)$ is even.

For the Davis manifold we have from [34] that $b_{1}(D)=24$ and $b_{2}(D)=72$. Since the signature of $D$ is zero, $b_{2}^{+}(D)=36$, and so the parity condition fails.

\section{3}

A natural weakening of Question 4.1 is the following.

Question 4.7 For $g, h \geq 2$, does there exist a short exact sequence:

$$
1 \rightarrow \pi_{1}\left(\Sigma_{g}\right) \rightarrow \Gamma \rightarrow \pi_{1}\left(\Sigma_{h}\right) \rightarrow 1
$$

for which $\Gamma$ is a word hyperbolic group?

Arguing as in the proof of Theorem 4.2, it follows that such an extension defines a purely pseudo-Anosov surface subgroup of $\Gamma_{g}$. However, even in this case, little is known. We make two comments in this regard.

1. One result is that $\Gamma$ cannot be the fundamental group of a closed complex hyperbolic surface. As described in [20], this follows from [25], on showing that if $\Gamma$ is as decribed, then there is a non-singular holomorphic fibration $X=\mathbf{H}_{\mathbf{C}}^{2} / \Gamma \rightarrow \Sigma_{h}$ that induces the short exact sequence (see also [17]). 
2. The following idea to construct purely pseudo-Anosov surface subgroups was described to me by Ian Agol. In [39], Thurston proves the following result (see [39] for terminology and further details).

Theorem 4.8 Let $n \geq 3$ and $k_{1}, k_{2} \ldots k_{n} \in(0,2 \pi)$ whose sum is $4 \pi$. Then the set of Euclidean cone metrics on $S^{2}$ with cone points of curvature $k_{i}$ and of total area 1 forms a complex hyperbolic manifold whose metric completion is a complex hyperbolic cone manifold of finite volume.

In addition, Thurston also gives conditions when such a cone manifold is an orbifold, and shows that certain of these completions give rise to a cocompact arithmetic lattices arising from Hermitian forms. These arithmetic lattices also arise in work of Mostow, and go back to Picard (see [10]). We will consider the example in [37] when $n=5$ and denote the lattice that Thurston constructs by $\Delta_{5}$. Since these arithmetic groups arise from Hermitian forms, it is well-known that they contain many cocompact Fuchsian subgroups; these can be R-Fuchsian, in the sense that they are subgroups of a group $\mathrm{SO}(2,1)$ or $\mathbf{C}$-Fuchsian in the sense that they are subgroups of $\mathrm{SU}(1,1)$.

Now if $\mathcal{M}_{0, n}$ denotes the moduli space of the $\mathrm{n}$-times punctured sphere, then Thurston shows that $\mathbf{H}_{\mathbf{C}}^{2} / \Delta_{5}$ is a compact orbifold corresponding to a compactification of $\mathcal{M}_{0,5}$. Agol has informed me that this compactification is the Mumford compactification. In terms of Thurston's description, the compactification locus is the locus at which pairs of cone points collide.

Question 4.9 Does there exist a cocompact Fuchsian subgroup of $\Delta_{5}$ that misses the compactification locus?

Given such a Fuchsian subgroup $F<\Delta_{5}$, Agol produces a purely pseudo-Anosov subgroup in some $\Gamma_{g}$ using a branched cover construction.

\section{4}

One generalization of looking for surface subgroups of $\Gamma_{g}$ is to look for injections of (cocompact) lattices in Lie groups into $\Gamma_{g}$. If the lattices are superrigid the image of such a lattice in $\Gamma_{g}$ is necessarily finite (see [12] and [42]), and so it follows (cf. Theorem 2 of [42]), that the only lattices that can admit a faithful representation (or even an infinite representation) into $\Gamma_{g}$ are lattices in $\mathrm{SO}(m, 1), m \geq 2$ or $\mathrm{SU}(q, 1), q \geq 1$. Indeed, since solvable subgroups of $\Gamma_{g}$ are virtually abelian [4], this observation also excludes non-cocompact lattices of $\mathrm{SU}(q, 1)$ for $q \geq 2$ from injecting. In addition, there is a simple obstruction to injecting certain of these lattices, or indeed for any group. Namely if a finitely generated group $G$ admits an injection into $\Gamma_{g}$, then $\operatorname{vcd}(G) \leq \operatorname{vcd}\left(\Gamma_{g}\right)$ (see [5]). If the vcd of a group $G$ satisfies the above inequality, then we call $G$ admissable. The vcd's of the groups $\Gamma_{g}$ are known to be $4 g-5$ when $g \geq 2$ [16]. Motivated by this discussion we pose.

Question 4.10 Let $\Gamma$ be a lattice in $\mathrm{SO}(m, 1), m \geq 3$ or $\mathrm{SU}(q, 1), q \geq 2$ which is admissable for $\Gamma_{g}$. Does $\Gamma$ inject in $\Gamma_{g}$ ? Can there be purely pseudo-Anosov representations?

If such an injection exists does there exist a continuous $\Gamma$-equivariant map

$$
\partial_{\infty}(\Gamma) \rightarrow \mathbb{P} \mathcal{M} \mathcal{L}_{0}(\Sigma) ?
$$

More generally, for a fixed $\Sigma$ and hence fixed vcd, a further natural generalization of the discussion here is:

Question 4.11 Which 1-ended admissable word hyperbolic groups $G$ inject in $\Gamma_{g}$ (as purely pseudoAnosov subgroups)?

If such an injection exists does there exist a continuous G-equivariant map

$$
\partial_{\infty}(G) \rightarrow \mathbb{P} \mathcal{M} \mathcal{L}_{0}(\Sigma) ?
$$


Remarks: 1. As discussed in [33], no example of a purely pseudo-Anosov non-free subgroup of $\Gamma_{g}$ is known at present.

2. It is a conjecture of Gromov that every 1-ended word hyperbolic group contains a surface subgroup (see [3]). Assuming this conjecture holds, then if there were any purely pseudo-Anosov injection of a 1-ended word hyperbolic group into $\Gamma_{g}$, this would produce a purely pseudo-Anosov surface subgroup of $\Gamma_{g}$.

3. As discussed in the Introduction, there are many analogies between non-uniform lattices in Lie groups and $\Gamma_{g}$. An analogy for purely pseduo-Anosov surface subgroup of $\Gamma_{g}$ would be a purely semisimple surface subgroup of a non-uniform lattice. In this regard, the groups $\operatorname{SL}(n, \mathbf{Z})$ for $n \geq 3$ all contain such surface groups. This can be seen be realizing certain arithmetic Fuchsian groups as subgroups of finite index in groups $\mathrm{SO}(f ; \mathbf{Z}) \subset \mathrm{SL}(n, \mathbf{Z})$, where $f$ is an indefinite ternary quadratic form with coefficients in $\mathbf{Z}$.

\section{References}

[1] I. Agol, D. D. Long and A. W. Reid, The Bianchi groups are separable on geometrically finite subgroups, Ann. of Math. 153 (2001), 599-621.

[2] M. F. Atiyah, The signature of fiber bundles, In Global Analysis (Papers dedicated to K. Kodaira), Princeton University Press, Princeton (1969), 73-84.

[3] M. Bestvina, Questions in geometric group theory, available at www.math.utah.edu/ bestvina.

[4] J. Birman, A. Lubotzky and J. McCarthy, Abelian and solvable subgroups of the mapping class groups, Duke Math. J. 50 (1983), 1107-1120.

[5] K. Brown, Cohomology of Groups, Graduate Texts in Math. 87 Springer-Verlag (1982).

[6] M. Conder and C. Maclachlan, Compact hyperbolic 4-manifolds of small volume, to appear Proc. A. M. S.

[7] D. Cooper and D. D. Long, Some surface subgroups survive surgery, Geom. Topol. 5 (2001), $347-367$.

[8] D. Cooper, D. D. Long and A. W. Reid, Essential surfaces in bounded 3-manifolds, J. A. M. S. 10 (1997), 553-563.

[9] M. W. Davis, A hyperbolic 4-manifold, Proc. A. M. S. 93 (1985), 325-328.

[10] P. Deligne and G. D. Mostow, Commensurabilities among lattices in PU $(1, n)$, Annals of Math. Studies, 132 Princeton University Press, Princeton, (1993).

[11] B. Farb, A. Lubotzky, and Y. Minsky, Rank-1 phenomena for mapping class groups, Duke Math. J. 106 (2001), 581-597.

[12] B. Farb and H. Masur, Superrigidity and mapping class groups, Topology 37 (1998), 11691176 .

[13] B. Farb and L. Mosher, Convex cocompact subgroups of mapping class groups, Geom. Topol. 6 (2002), 91-152. 
[14] R. E. Gompf and A. I. Stipsicz, 4-Manifolds and Kirby Calculus, Graduate Studies in Mathematics, 20 American Mathematical Society, (1999).

[15] G. Gonzalez-Diez and W. J. Harvey, Surface groups in Mapping Class groups, Topology 38 (1999), 57-69.

[16] J. L. Harer, The cohomology of the moduli space of curves, Theory of moduli (Montecatini Terme, 1985), 138-221, Lecture Notes in Math., 1337, Springer-Verlag (1988).

[17] J. A. Hillman, Complex surfaces which are fibre bundles, Topology and its Appl. 100 (2000), $187-191$

[18] N. V. Ivanov, Subgroups of Teichmüller modular groups, Transl. Math. Monogr., 115, Am. Math. Soc., Providence, RI, 1992.

[19] R. P. Kent and C. J. Leininger, Shadows of Mapping Class groups: capturing convex cocompactness, in preparation.

[20] M. Kapovich, On normal subgroups in the fundamental groups of complex surfaces, preprint.

[21] C. LeBrun, Hyperbolic manifolds, harmonic forms and Seiberg-Witten invariants, Proceedings of the Euroconference on Partial Differential Equations and their Applications to Geometry and Physics (Castelvecchio Pascoli, 2000). Geom. Dedicata 91 (2002), 137-154.

[22] C. J. Leininger, On groups generated by two positive multi-twists: Teichmüller curves and Lehmer's number, Geometry and Topology 8 (2004), 1301-1359.

[23] C. J. Leininger and A. W. Reid, A combination theorem for Veech subgroups of the mapping class group, preprint, arXiv:math.GT/0410041.

[24] C. J. Leininger and D. B. McReynolds, Separable subgroups of the Mapping class group, in preparation.

[25] K. Liu, Geometric height inequalities, Math. Research Letters 3 (1996), 693-702.

[26] B. Maskit, Kleinian groups, Springer-Verlag Berlin Heidelberg, 1988.

[27] J. D. Masters and X. Zhang, private communication.

[28] H. Masur, Two boundaries of Teichmüller space, Duke Math. J. 49 (1982), 183-190.

[29] J. D. McCarthy, On the first cohomology of cofinite subgroups in surface mapping class groups, Topology 40 (2001), 401-418.

[30] J. McCarthy and A. Papadopoulos, Dynamics on Thurston's sphere of projective measured foliations, Comment. Math. Helv. 64 (1989), 133-166.

[31] C. T. McMullen, Billiards and Teichmüller curves on Hilbert Modular surfaces, J. A. M. S. 16 (2003), 857-885.

[32] J. W. Morgan, On Thurston's uniformization theorem for three-dimensional manifolds, The Smith conjecture (New York, 1979), 37-125, Pure Appl. Math., 112, Academic Press, (1984).

[33] L. Mosher, Problems in the geometry of surface group extensions, preprint. 
[34] J. Ratcliffe and S. Tschantz, On the Davis hyperbolic 4-manifold, Topology and its Appl. 111 (2001), 327-342.

[35] G. P. Scott, Subgroups of surface groups are almost geometric, J. London Math. Soc. 17 (1978), 555 - 565. See also ibid Correction: J. London Math. Soc. 32 (1985), 217-220.

[36] C. H. Taubes, The Seiberg-Witten invariants and symplectic forms, Math. Research Letters 1 (1994), 809-822.

[37] W. P. Thurston, The Geometry and Topology of Three-Manifolds, Princeton University course notes, available at http://www.msri.org/publications/books/gt3m/ (1980).

[38] W. P. Thurston, Some simple examples of symplectic manifolds, Proc. A. M. S. 55 (1976), $467-468$.

[39] W. P. Thurston, Shapes of polyhedra and triangulations of the sphere, Geometry and Topology Monographs, 1 The Epstein Birthday Schrift, eds. I. Rivin, C. Rourke, and C. Series 511-549 (1998).

[40] W. A. Veech, Teichmüller curves in moduli space, Eisenstein series and an application to triangular billiards, Invent. Math. 97 (1989), 553-583.

[41] H. C. Wang, Topics in totally discontinuous groups, in Symmetric Spaces, eds. W. M. Boothby and G. L. Weiss, Pure and Appl. Math 8, Marcel Dekker (1972), pp 459-487.

[42] S-K. Yeung, Representations of semisimple lattices in Mapping class groups, International Math. Research Notices 31 (2003), 1677-1686.

Department of Mathematics,

University of Texas

Austin, TX 78712 